\title{
On the mechanism of integration of informatization and industrialization based on dynamics of stochastic model
}

\author{
Chaodong Yan*, Jing Ma, Dandan Li \\ College of Economics and Management, Nanjing University of Aeronautics and Astronautics, Nanjing 211106, China.
}

Communicated by A. Atangana

\begin{abstract}
In recent years, with the new generation of information and communication technology in the industrial field more widely used, the integration of informatization and industrialization aroused people's research and attention again. The connotation and mechanism of integration of informatization and industrialization is one of the focuses of people's research. In this paper, a stochastic model that describes the interaction between information and industrial technology is proposed and analyzed. Sharp sufficient conditions for increase and stagnate of the diffusion rates for both information and industrial technology are established. The results reveal that the stochastic perturbations can affect the dynamics of model significantly. (C)2017 All rights reserved.
\end{abstract}

Keywords: Integration of the informatization and industrialization, stochastic perturbations, information technology, industrial technology, increase, stagnate.

2010 MSC: 60H10, 60H30.

\section{Introduction}

To address problems arising the process of industrialization such as large resource consumption, heavy pollution, low technological, content and insufficient economic benefits, the Chinese government has proposed the strategy of "reciprocal promotion between industrialization and informatization" since the late 1990s. Subsequently, "striving for the integration of informatization and industrialization" and "further facilitating the deep integration of informatization and industrialization" were presented at the 17th and 18th Congresses of the CPC. To now, China has scored remarkable accomplishments regarding the integration of informatization and industrialization as practices and explorations proceed, paving China's path to speedy industrial modernization.

In recent years, informatization has recorded profound influence on socioeconomic development amid rapid progress and wide application of new communication technologies such as cloud computing, big data, Internet of things and mobile Internet. Developed countries including America, Germany, and Japan have implemented a "re-industrialization" strategy centering on integrated application of new communication technologies to industrial field, in a bid to cultivate new advantages in national competition

\footnotetext{
*Corresponding author

Email address: yanchaodong@163.com (Chaodong Yan)
} 
and capture the commanding height of industrial development. China has put strategies of "Made in China 2025" and "Internet+" into practice in 2015, arousing again public concerns over the integration of informatization and industrialization.

Recent research of informatization and industrialization $[1,3,4,6,8,9,17,20,22,25,27,28]$ primarily focuses on: the connotation and mechanism of integration of informatization and industrialization, the relationship between informatization and industrialization, particularly the role of informatization in industrialization, the coupling coordination between informatization and industrialization, the measurement of integration of industrialization and informatization, and the path to integration of informatization and industrialization, as well as suggestions on policies. Previous studies have scored remarkable achievements, providing a valuable reference for further research. Yet definitely their studies are not perfect in default of consensus on and mathematical supports for the connotation and mechanism of integration of informatization and industrialization.

Further study on the connotation and mechanism of integration of informatization and industrialization is in demand, for they represent the logical starting and theoretical basis for relevant research, policies making and implementing. Below are representative views concerning the content of integration of informatization and industrialization. Wu [24] deems that reciprocal promotion between informatization and industrialization constitutes the essence of the integration. According to Jin [10], integration of informatization and industrialization is all-round, multi-level, trans-regional and integrated. Informatization is incorporated into several processes such as procurement, design, production, sales, and customer service in four respects, i.e., technology integration, product integration, business integration and industry derivation. In Miao's opinion [19], integration of informatization and industrialization broadly signifies the integration of two historical processes. And in a narrow sense, it is the process of industrial restructuring and upgrading resulting from tweaks in production efficiency and mode when information technology is widely applied to industry. As noted by Wang [21], integration of informatization and industrialization is indeed a form of information technology leapfrogging, that is, tipping from inefficient types of technology directly to higher-efficiency ones, with certain intermediate stages bypassed. Xie et al. [26] identified integration of informatization and industrialization as a superposition and evolution process in which informatization and industrialization exert influence on each other, thus in essence, it is a convergence phenomenon. Integration of informatization and industrialization stresses informatization of industrial production, claims Wang and Du [23], underscoring integration of information technology into product design, manufacturing, marketing and other chains. Though opinions are divided in the content of integration of informatization and industrialization, a consensus is underway, that is, integration of informatization and industrialization highlights reciprocal promotion between informatization and industrialization, thereby achieving common progress.

To clarify the mechanism of integration of informatization and industrialization, this paper regards the integration as a number of successive technological life cycles in virtue of theories of technological leapfrogging and technological life cycle. Integration of informatization and industrialization signifies the process of technological leapfrogging in one technological life cycle. On the one hand, the information technology of this life cycle is widely used in industrial productive process, integrated with original industrial technology, business process and production mode, which formed a more innovative and diffusivity productivity, and it makes the business process optimize, production mode change and productive efficiency enhance. When this productive paradigm permeates the entire industrial sector, it can promote the industrial technological progress, structural adjustment and transformation upgrading. On the other hand, factors such as industrial technological progress and market competition also put forward higher request to information technology which promotes the further development of information technology. Furthermore, when the higher level of information technology is applied to social life and other national economic field, it promotes the information level of the whole society at the same time.

In the above part, we present a theoretical description of the connotation and mechanism of integration of informatization and industrialization. Vivid as it is, it lacks mathematical supports for further fullfledged and convincing demonstration. Wang [21] provides a favorable model for reference in this respect. 
Yet he adopted dynamics of conventional model, which neglected the effects of external stochastic factors such as market and policy on the integration of informatization and industrialization. It is unreasonable, in reality, to neglect the effects of external factors as the integration will definitely subject to government guidance, market competition, financial policy, and other external factors. By establishing dynamics of stochastic model taking external factors into account, succeeding parts of this paper will specify the influence of noise disturbance on integration of informatization and industrialization.

\section{Mathematical model}

One of the famous frameworks for modeling the interactions between the information technology and industrial technology is the following model ([21]):

$$
\left\{\begin{array}{l}
\frac{d f_{1}(t)}{d t}=f_{1}(t)\left[\alpha_{1}-f_{1}(t)+\beta_{1} f_{2}(t)\right] \\
\frac{d f_{2}(t)}{d t}=f_{2}(t)\left[\alpha_{2}-f_{2}(t)+\beta_{2} f_{1}(t)\right]
\end{array}\right.
$$

where $f_{1}(t)$ stands for the diffusion rate of information technology $\left(T_{1}\right)$, and $f_{2}(t)$ is the diffusion rate of industrial technologies $\left(T_{2}\right)$ after the introduction of information technology. $\alpha_{1}$ and $\alpha_{2}$ are the growth rates of the information technology and the industrial technologies, respectively. $\alpha_{1}$ and $\alpha_{2}$ measure the strengths of investments on $T_{1}$ and $T_{2}$, respectively. $\beta_{1}$ and $\beta_{2}$ are the influence rates of $T_{2}$ to $T_{1}$ and $T_{1}$ to $T_{2}$, respectively, $0 \leqslant \beta_{1}, \beta_{2} \leqslant 1$ (because the influence rates between $T_{1}$ and $T_{2}$ are smaller than the self-influence rates of $T_{1}$ and $T_{2}$ ).

Now let us take the environmental perturbations into account. As said above, the growth of the information technology and the industrial technologies in the real world are often affected by stochastic perturbations, for example, the fluctuation of the economic development. Recall that $\alpha_{1}$ and $\alpha_{2}$ are the growth rates of $T_{1}$ and $T_{2}$, respectively. In practice one always estimates $\alpha_{i}$ by an average value plus an error term. Generally, according to the central limit theorem, the error term follows a normal distribution. Consequently, for a short correlation time, $\alpha_{i}$ can be replaced by

$$
\alpha_{i}+\gamma_{i} \dot{B}_{i}(t)
$$

Here we still use $\alpha_{i}$ to represent the average growth rate; $\gamma_{i}^{2}$ is the intensity of the random perturbation; and $\left\{\mathrm{B}_{1}(\mathrm{t})\right\}_{\mathrm{t} \geqslant 0}$ and $\left\{\mathrm{B}_{2}(\mathrm{t})\right\}_{\mathrm{t} \geqslant 0}$ are two standard Brownian motions defined on a complete probability space $\left(\Omega,\left\{\mathcal{F}_{t}\right\}_{t} \geqslant 0, P\right)([12,14])$. Thus system $(2.1)$ becomes

$$
\left\{\begin{array}{l}
\left.d f_{1}(t)=f_{1}(t)\left[\alpha_{1}-f_{1}(t)+\beta_{1} f_{2}(t)\right)\right] d t+\gamma_{1} f_{1}(t) d B_{1}(t) \\
\left.d f_{2}(t)=f_{2}(t)\left[\alpha_{2}-f_{2}(t)+\beta_{2} f_{1}(t)\right)\right] d t+\gamma_{2} f_{2}(t) d B_{2}(t) .
\end{array}\right.
$$

\section{Increase and stagnation}

To begin with, let us define some notations and prepare some lemmas:

$$
\begin{aligned}
\mathbb{R}_{+}^{2} & =\left\{a=\left(a_{1}, a_{2}\right) \in \mathbb{R}^{2} \mid a_{i}>0, i=1,2\right\}, \theta_{i}=0.5 \gamma_{i}^{2}, i=1,2, \\
b_{i} & =\alpha_{i}-\gamma_{i}^{2} / 2, \Delta=1-\beta_{1} \beta_{2}, \Delta_{1}=b_{1}+b_{2} \beta_{1}, \Delta_{2}=b_{2}+b_{1} \beta_{2}, \\
\Gamma & =\theta_{1} \alpha_{2}-\theta_{2} \alpha_{1}, C_{1}=\alpha_{1}+\beta_{1} \alpha_{2}, C_{2}=\alpha_{2}+\beta_{2} \alpha_{1} \\
D_{1} & =\theta_{1}+\beta_{1} \theta_{2}, D_{2}=\theta_{2}+\beta_{2} \theta_{1}, \xi_{1}=\alpha_{1} / \theta_{1}, \xi_{2}=C_{2} / D_{2}, \\
\overline{g(t)} & =t^{-1} \int_{0}^{t} g(s) d s, g^{*}=\limsup _{t \rightarrow+\infty} g(t), g_{*}=\liminf _{t \rightarrow+\infty} g(t) .
\end{aligned}
$$


Clearly, $b_{i}=\alpha_{i}-\theta_{i}, \Delta_{i}=C_{i}-D_{i}, i=1,2$. Throughout this paper, without loss of generality, we always suppose that $\alpha_{1} / \theta_{1}>\alpha_{2} / \theta_{2}$. Hence $\xi_{1} \geqslant \xi_{2}$.

Note that $f_{1}(t)$ and $f_{2}(t)$ are the diffusion rates, then we must have $f_{1}(t)>0$ and $f_{2}(t)>0$ to be realistic.

Lemma 3.1. For any given initial condition $\mathrm{f}(0) \in \mathbb{R}_{+}^{2}$, system (2.2) has a unique global positive solution $\mathrm{f}(\mathrm{t})=$ $\left(\mathrm{f}_{1}(\mathrm{t}), \mathrm{f}_{2}(\mathrm{t})\right)^{\mathrm{T}}$ almost surely (a.s.). Moreover, for any $\mathrm{p}>1$, there is a positive constant $\mathrm{K}=\mathrm{K}(\mathrm{p})$ such that

$$
\limsup _{t \rightarrow+\infty} \mathbb{E}\left(f_{i}^{p}(t)\right) \leqslant K .
$$

Proof. Note that $\Delta=1-\beta_{1} \beta_{2}>0$, hence there are two constants $c_{1}>0$ and $c_{2}>0$ such that

$$
-2 a:=\lambda_{\max }\left(C \beta+\beta^{\top} C\right)<0,
$$

where

$$
\beta=\left(\begin{array}{cc}
-1 & \beta_{1} \\
\beta_{2} & -1
\end{array}\right), C=\left(\begin{array}{cc}
c_{1} & 0 \\
0 & c_{2}
\end{array}\right),
$$

and $\lambda_{\max }\left(C \beta+\beta^{\top} C\right)$ is the largest eigenvalue of $C \beta+\beta^{\top} C$. As a matter of fact, there are $c_{1}>0$ and $c_{2}>0$ such that $\beta_{1} c_{1}=\beta_{2} c_{2}$, then

$$
\left|C \beta+\beta^{\top} C\right|=4 c_{1} c_{2} \Delta>0 .
$$

Hence,

$$
\lambda_{\max }\left(C \beta+\beta^{T} C\right)<0 .
$$

Define

$$
V(f)=c_{1} f_{1}+c_{2} f_{2}, f \in \mathbb{R}_{+}^{2} .
$$

It then follows from Itô's formula ([18]) that

$$
\begin{aligned}
d V(f) & =c_{1} f_{1}\left[\alpha_{1}-f_{1}-\beta_{1} f_{2}\right] d t+c_{2} f_{2}\left[\alpha_{2}-f_{2}-\beta_{2} f_{1}\right] d t+c_{1} \gamma_{1} f_{1} d B_{1}(t)+c_{2} \gamma_{2} f_{2} d B_{2}(t) \\
& =\left[c_{1} \alpha_{1} f_{1} \alpha_{1}+c_{2} \alpha_{2} f_{2}+f^{\top} C \beta f\right] d t+c_{1} \gamma_{1} f_{1} d B_{1}(t)+c_{2} \gamma_{2} f_{2} d B_{2}(t) \\
& =\left[c_{1} \alpha_{1} f_{1} \alpha_{1}+c_{2} \alpha_{2} f_{2}+\frac{1}{2} f^{\top}\left(C \beta+\beta^{\top} C\right) f\right] d t+c_{1} \gamma_{1} f_{1} d B_{1}(t)+c_{2} \gamma_{2} f_{2} d B_{2}(t) \\
& \leqslant\left[c_{1} \alpha_{1} f_{1} \alpha_{1}+c_{2} \alpha_{2} f_{2}-a\left(f_{1}^{2}+f_{2}^{2}\right)\right] d t+c_{1} \gamma_{1} f_{1} d B_{1}(t)+c_{2} \gamma_{2} f_{2} d B_{2}(t) \\
& \leqslant k_{1} d t+c_{1} \gamma_{1} f_{1} d B_{1}(t)+c_{2} \gamma_{2} f_{2} d B_{2}(t) .
\end{aligned}
$$

The following proof is similar to these in [15], and we omit them.

Lemma 3.2. For model (2.2), we have

$$
\limsup _{t \rightarrow+\infty} \frac{\ln f_{i}(t)}{\ln t} \leqslant 1, \text { a.s., } i=1,2 \text {. }
$$

Proof. An application of Itô's formula to $e^{t} V(f)=e^{t}\left(c_{1} f_{1}+c_{2} f_{2}\right)$ leads to

$$
\begin{aligned}
d\left[e^{t} V(f(t))\right]= & e^{t} V(f(t)) d t+e^{t} d V(f(t)) \\
\leqslant & e^{t}\left[c_{1} f_{1}(t)+c_{2} f_{2}(t)+\alpha_{1} c_{1} f_{1}(t)+\alpha_{2} c_{2} f_{2}(t)-a\left(f_{1}^{2}(t)+f_{2}^{2}(t)\right)\right] d t \\
& +e^{t}\left[c_{1} \gamma_{1} f_{1}(t) d B(t)+c_{2} \gamma_{2} f_{2}(t) d B(t)\right] \\
\leqslant & k_{1} e^{t} d t+e^{t}\left[c_{1} \gamma_{1} f_{1}(t) d B(t)+c_{2} \gamma_{2} f_{2}(t) d B(t)\right] .
\end{aligned}
$$


Therefore,

$$
\limsup _{t \rightarrow+\infty} \mathbb{E V}(f(t)) \leqslant k_{1}
$$

Note that $|f| \leqslant f_{1}+f_{2} \leqslant V(f) / \min \left\{c_{1}, c_{2}\right\}$, hence

$$
\limsup _{t \rightarrow+\infty} \mathbb{E}|f(t)| \leqslant k_{1} / \min \left\{c_{1}, c_{2}\right\}=: k_{2} .
$$

Moreover, according to Itô's formula,

$$
\begin{aligned}
\mathbb{E V}(f(t+1)) & \leqslant \mathbb{E V}(f(t))+\mathbb{E} \int_{t}^{t+1}\left[\alpha_{1} c_{1} f_{1}(s)+\alpha_{2} c_{2} f_{2}(s)-a\left(f_{1}^{2}(s)+f_{2}^{2}(s)\right)\right] d s \\
& \leqslant \mathbb{E V}(f(t))+q \mathbb{E} \int_{t}^{t+1}|f(s)| d s-a \mathbb{E} \int_{t}^{t+1}|f(s)|^{2} d s
\end{aligned}
$$

where $q=\sqrt{2} \max \left\{\alpha_{1} c_{1}, \alpha_{2} c_{2}\right\}$. It then follows from $\mathbb{E V}(f(t+1)) \geqslant 0$ that

$$
\limsup _{t \rightarrow+\infty} \mathbb{E} \int_{t}^{t+1}|f(s)|^{2} d s \leqslant\left(k_{1}+q k_{2}\right) / a=: k_{3} .
$$

An application of Itô's formula again yields

$$
\begin{aligned}
\mathbb{E}\left(\sup _{t \leqslant u \leqslant t+1} V(f(u))\right) \leqslant & \mathbb{E V}(f(t))+q \mathbb{E} \int_{t}^{t+1}|f(s)| d s+c_{1} \gamma_{1} \mathbb{E}\left(\sup _{t \leqslant u+t+1}\left|\int_{t}^{u} f_{1}(s) d B_{1}(s)\right|\right) \\
& +c_{2} \gamma_{2} \mathbb{E}\left(\sup _{t \leqslant u \leqslant t+1}\left|\int_{t}^{u} f_{2}(s) d B_{2}(s)\right|\right) .
\end{aligned}
$$

Let

$$
M_{1}(t)=\int_{t}^{u} f_{1}(s) d B_{1}(s), M_{2}(t)=\int_{t}^{u} f_{2}(s) d B_{2}(s) .
$$

According to the Burkholder-Davis-Gundy inequality and the Hölder inequality, one has

$$
\begin{aligned}
& \mathbb{E}\left(\sup _{t \leqslant u \leqslant t+1}\left|M_{1}(u)\right|\right) \leqslant k_{4} \mathbb{E}\left(\int_{t}^{t+1} f_{1}^{2}(s) d s\right)^{0.5} \leqslant k_{4}\left(\mathbb{E} \int_{t}^{t+1} f_{1}^{2}(s) d s\right)^{0.5} \leqslant k_{4}\left(\mathbb{E} \int_{t}^{t+1}|f(s)|^{2} d s\right)^{0.5}, \\
& \mathbb{E}\left(\sup _{t \leqslant u \leqslant t+1}\left|M_{2}(u)\right|\right) \leqslant k_{4}\left(\mathbb{E} \int_{t}^{t+1} f_{2}^{2}(s) d s\right)^{0.5} \leqslant k_{4}\left(\mathbb{E} \int_{t}^{t+1}|f(s)|^{2} d s\right)^{0.5},
\end{aligned}
$$

where $k_{4}>0$ is a constant. When these two inequalities are used in (3.6) and note that (3.3), (3.4), and (3.5) hold, then we have

$$
\limsup _{t \rightarrow+\infty} \mathbb{E}\left(\sup _{t \leqslant u \in t+1} V(f(u))\right) \leqslant k_{1}+q k_{2}+\left[c_{1} \gamma_{1} k_{4}+c_{2} \gamma_{2} k_{4}\right] k_{3}^{0.5} .
$$

Consequently,

$$
\mathbb{E}\left(\sup _{n \leqslant u \leqslant n+1}|f(u)|\right) \leqslant k_{5}, n=1,2, \ldots,
$$

where $k_{5}>0$ is a constant. For arbitrary $\varepsilon>0$, by Chebyshev's inequality, we obtain

$$
P\left\{\sup _{n \leqslant t \leqslant n+1}|f(t)|>k^{1+\varepsilon}\right\} \leqslant \frac{k_{5}}{k^{1+\varepsilon}}, n=1,2, \ldots
$$

According to the Borel-Cantelli lemma, there exists an $n_{0}$ such that for almost all $\omega \in \Omega$, if $n \geqslant n_{0}$ and 
$\mathrm{n} \leqslant \mathrm{t} \leqslant \mathrm{n}+1$,

$$
\sup _{n \leqslant t \leqslant n+1}|f(t)| \leqslant n^{1+\varepsilon}
$$

In other words,

$$
\frac{\ln |f(t)|}{\ln t} \leqslant \frac{(1+\varepsilon) \ln n}{\ln n}=1+\varepsilon .
$$

Letting $\varepsilon \rightarrow 0$, we obtain the required assertion.

Lemma 3.3 ([16]). Suppose that $\mathrm{Z}(\mathrm{t}) \in \mathrm{C}\left(\Omega \times[0,+\infty), \mathbb{R}_{+}\right)$.

(I) If there exist two positive constants $T$ and $\delta_{0}$ such that

$$
\ln Z(t) \leqslant \delta t-\delta_{0} \int_{0}^{t} Z(s) d s+\sum_{i=1}^{2} \tau_{i} B_{i}(t), \quad \text { a.s. }
$$

for all $\mathrm{t} \geqslant \mathrm{T}$, where $\tau_{i}, \delta$, and $\delta_{0}$ are constants, then

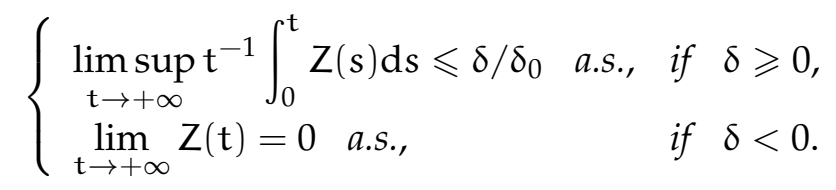

(II) If there exist three positive constants $\mathrm{T}, \delta$, and $\delta_{0}$ such that

$$
\ln Z(t) \geqslant \delta t-\delta_{0} \int_{0}^{t} Z(s) d s+\sum_{i=1}^{2} \tau_{i} B_{i}(t), \text { a.s. }
$$

for all $\mathrm{t} \geqslant \mathrm{T}$, then $\liminf _{\mathrm{t} \rightarrow+\infty} \mathrm{t}^{-1} \int_{0}^{\mathrm{t}} \mathrm{Z}(\mathrm{s}) \mathrm{d} \mathrm{s} \geqslant \delta / \delta_{0}$, a.s..

Lemma 3.4. For model (2.2),

(i) If $1<\xi_{2}$, then

$$
\lim _{t \rightarrow+\infty} t^{-1} \int_{0}^{t} f_{1}(s) d s=\frac{\Delta_{1}}{\Delta}, \quad \lim _{t \rightarrow+\infty} t^{-1} \int_{0}^{t} f_{2}(s) d s=\frac{\Delta_{2}}{\Delta}, \text { a.s.. }
$$

(ii) If $\xi_{2}<1<\xi_{1}$, then $\lim _{\mathrm{t} \rightarrow+\infty} f_{2}(\mathrm{t})=0$ a.s. and

$$
\lim _{t \rightarrow+\infty} t^{-1} \int_{0}^{t} f_{1}(s) d s=b_{1} \text {, a.s.. }
$$

(iii) If $\xi_{1}<1$, then $\lim _{t \rightarrow+\infty} f_{i}(t)=0$ a.s., $i=1,2$.

Proof. An application of Itô's formula leads to

$$
\begin{aligned}
& \ln f_{1}(t)-\ln f_{1}(0)=b_{1} t-\int_{0}^{t} f_{1}(s) d s-\beta_{1} \int_{0}^{t} f_{2}(s) d s+\gamma_{1} B_{1}(t) \\
& \ln f_{2}(t)-\ln f_{2}(0)=b_{2} t-\beta_{2} \int_{0}^{t} f_{1}(s) d s-\int_{0}^{t} f_{2}(s) d s+\gamma_{2} B_{2}(t) .
\end{aligned}
$$

It is easy to see that

$$
\xi_{1}=\alpha_{1} / \theta_{1} \geqslant \xi_{2}=C_{2} / D_{2}
$$


Compute that (3.7) $-(3.8) \times \beta_{1}$, one can obtain that

$$
t^{-1} \ln \left(f_{1}(t) / f_{1}(0)\right)-\beta_{1} t^{-1} \ln \left(f_{2}(t) / f_{2}(0)\right)=C_{1}-D_{1}-\Delta \overline{f_{1}(t)}+t^{-1}\left[\gamma_{1} B_{1}(t)-\beta_{1} \gamma_{2} B_{2}(t)\right] .
$$

In the same way, compute (3.8)-(3.7) $\times \beta_{2}$, we get

$$
t^{-1} \ln \left(f_{2}(t) / f_{2}(0)\right)-t^{-1} \beta_{2} \ln \left(f_{1}(t) / f_{1}(0)\right)=C_{2}-D_{2}-\Delta \overline{f_{2}(t)}+t^{-1}\left[\gamma_{2} B_{2}(t)-\beta_{2} \gamma_{1} B_{1}(t)\right] .
$$

According to (3.7) and (3.8), for sufficiently large $t$,

$$
\begin{aligned}
& \frac{\ln \left(f_{1}(t) / f_{1}(0)\right)}{t} \leqslant b_{1}+\varepsilon-\overline{f_{1}(t)}-\beta_{1}{\overline{f_{2}}}^{*}+\gamma_{1} B_{1}(t) / t \\
& \frac{\ln \left(f_{2}(t) / f_{2}(0)\right)}{t} \leqslant b_{2}+\varepsilon-\beta_{2} \bar{f}_{1}{ }^{*}-\overline{f_{2}(t)}+\gamma_{2} B_{2}(t) / t .
\end{aligned}
$$

Define

$$
\phi_{1}=b_{1}+\varepsilon-\beta_{1}{\overline{f_{2}}}^{*}, \quad \phi_{2}=b_{2}+\varepsilon-\beta_{2}{\overline{f_{1}}}^{*} .
$$

Consequently,

$$
\begin{aligned}
& \frac{\ln \left(f_{1}(t) / f_{1}(0)\right)}{t} \leqslant \phi_{1}-\overline{f_{1}(t)}+\gamma_{1} B_{1}(t) / t \\
& \frac{\ln \left(f_{2}(t) / f_{2}(0)\right)}{t} \leqslant \phi_{2}-\overline{f_{2}(t)}+\gamma_{2} B_{2}(t) / t .
\end{aligned}
$$

(i). According to (3.2), for arbitrarily $\varepsilon>0$, there is a $T>0$ such that for all $t \geqslant T$

$$
-\beta_{1} t^{-1} \ln \left(f_{2}(t) / f_{2}(0)\right) \leqslant-\beta_{1}\left[t^{-1} \ln f_{2}\right]^{*}+\varepsilon \leqslant \varepsilon .
$$

When this inequality is used in (3.9), one can see that

$$
t^{-1} \ln \left(f_{1}(t) / f_{1}(0)\right) \geqslant C_{1}-D_{1}-\varepsilon-\Delta \overline{f_{1}(t)}+t^{-1}\left[\gamma_{1} B_{1}(t)-\beta_{1} \gamma_{2} B_{2}(t)\right]
$$

Note that $C_{1} / D_{1} \geqslant C_{2} / D_{2}>1$, hence we can choose $\varepsilon$ sufficiently small such that $C_{1}-D_{1}-\varepsilon>0$. It then follows from (II) in Lemma 3.3 and the arbitrariness of $\varepsilon$ that

$$
\overline{\mathrm{f}_{1 *}} \geqslant \frac{\mathrm{C}_{1}-\mathrm{D}_{1}}{\Delta}=\frac{\Delta_{1}}{\Delta}, \text { a.s.. }
$$

Hence $\phi_{1}>0$. Similarly, an application of (3.10) gives

$$
\overline{\mathrm{f}_{2 *}} \geqslant \frac{\Delta_{2}}{\Delta}
$$

Thereby $\phi_{2}>0$. According to (I) in Lemma 3.3, one can observe that

$$
{\overline{\mathrm{f}_{1}}}^{*} \leqslant \phi_{1},{\overline{\mathrm{f}_{2}}}^{*} \leqslant \phi_{2} .
$$

In other word,

$$
{\overline{f_{1}}}^{*}+\beta_{1}{\overline{f_{2}}}^{*} \leqslant b_{1}+\varepsilon, \beta_{2}{\overline{f_{1}}}^{*}+{\overline{f_{2}}}^{*} \leqslant b_{2}+\varepsilon, \text { a.s.. }
$$

Therefore

$$
{\overline{f_{1}}}^{*} \leqslant \Delta_{1} / \Delta,{\overline{f_{2}}}^{*} \leqslant \Delta_{2} / \Delta, \text { a.s.. }
$$

Then we obtain the desired assertion. 
(ii). Note that $C_{1} / D_{1}>1$, hence (3.13) holds. In other words,

$$
\overline{\mathrm{f}_{1 *}}>\Delta_{1} / \Delta \text {. }
$$

Then $\phi_{1}>0$, and hence (3.14) holds. If $\omega \in\left\{{\overline{f_{2}(\omega)}}^{*}>0\right\}$, in view of Lemma 3.3, we get

$$
{\overline{f_{2}(\omega)}}^{*} \leqslant \phi_{2}=b_{2}+\varepsilon-\beta_{2}{\overline{f_{1}(\omega)}}^{*} .
$$

When this inequality is used in (3.14), we get

$$
0<\Delta{\overline{f_{2}(\omega)}}^{*} \leqslant b_{2}-\beta_{2} b_{1}+\varepsilon-\beta_{2} \varepsilon=C_{2}-D_{2}+\varepsilon-\beta_{2} \varepsilon .
$$

An application of the arbitrariness of $\varepsilon$ gives $C_{2} / D_{2} \geqslant 1$. Then the contradiction arises. Thereby,

$$
\mathcal{P}\left\{\omega:{\overline{f_{2}}}^{*}>0\right\}=0,
$$

that is to say,

$$
{\overline{\mathrm{f}_{2}}}^{*}=0 \text {, a.s.. }
$$

Substituting (3.14) into (3.12) results in

$$
\begin{aligned}
\frac{\ln \left(f_{2}(t) / f_{2}(0)\right)}{t} & \leqslant b_{2}+\varepsilon-\beta_{2}\left(b_{1}+\varepsilon-\beta_{1}{\overline{f_{2}}}^{*}\right)-\overline{f_{2}(t)}+\gamma_{2} B_{2}(t) / t \\
& =C_{2}-D_{2}+\varepsilon(t)+\varepsilon-\beta_{2} \varepsilon+\gamma_{2} B_{2}(t) / t
\end{aligned}
$$

where $\varepsilon(t)=\beta_{1} \beta_{2}{\overline{f_{2}}}^{*}-\overline{f_{2}(t)}$. Note that $1>C_{2} / D_{2}$, hence ${\overline{f_{2}}}^{*}=0$. Therefore $\varepsilon(t) \rightarrow 0$. By virtue of Lemma 3.3,

$$
\lim _{t \rightarrow+\infty} f_{2}(t)=0, \text { a.s.. }
$$

According to (3.7), for sufficiently large $t$, one can see that

$$
\begin{aligned}
& t^{-1} \ln \frac{f_{1}(t)}{f_{1}(0)} \leqslant b_{1}+\varepsilon-\overline{f_{1}(t)}+\gamma_{1} B_{1}(t) / t \\
& t^{-1} \ln \frac{f_{1}(t)}{f_{1}(0)} \geqslant b_{1}-\varepsilon-\overline{f_{1}(t)}+\gamma_{1} B_{1}(t) / t
\end{aligned}
$$

where $\varepsilon \in\left(0, b_{1}\right)$. Applying (I) and (II) in Lemma 3.3 to (3.15) and (3.16), respectively, one can see that

$$
b_{1}-\varepsilon \leqslant \overline{f_{1 *}} \leqslant \overline{f_{1}} \leqslant b_{1}+\varepsilon \text {, a.s.. }
$$

It then follows from the arbitrariness of $\varepsilon$ that

$$
\lim _{t \rightarrow+\infty} \overline{f_{1}(t)}=b_{1} \text {, a.s.. }
$$

(iii). If ${\overline{f_{1}}}^{*}>0$, then $\phi_{1}>0$. Similar to the proof of (ii), one can see that $\lim _{t \rightarrow+\infty} f_{2}(t)=0$, a.s..

If ${\overline{f_{1}}}^{*}=0$, by (3.12), for sufficiently large $t$,

$$
\frac{\ln \left(f_{2}(t) / f_{2}(0)\right)}{t} \leqslant b_{2}+\varepsilon-\overline{f_{2}(t)}+\gamma_{2} B_{2}(t) / t .
$$

Note that $1>C_{2} / D_{2}>\alpha_{2} / \beta_{2}$, and in view of Lemma 3.3, we have $\lim _{t \rightarrow+\infty} f_{2}(t)=0$, a.s..

In the above, we have proved that $\lim _{t \rightarrow+\infty} f_{2}(t)=0$, a.s.. According to (3.11), for sufficiently large $t$,

$$
\frac{\ln \left(f_{1}(t) / f_{1}(0)\right)}{t} \leqslant b_{1}+\varepsilon-\overline{f_{1}(t)}+\gamma_{1} B_{1}(t) / t .
$$

By $1>\alpha_{1} / \beta_{1}$ and Lemma 3.3, we obtain the required assertion. 
Lemma 3.5. Model (2.2) is asymptotically stable in distribution, i.e., there is a unique probability measure $\mu(\cdot)$ such that for arbitrary $\mathrm{f}(0) \in \mathbb{R}_{+}^{2}$, the transition probability $\mathrm{p}(\mathrm{t}, \mathrm{f}(0), \cdot)$ of $\mathrm{f}(\mathrm{t})$ converges to $\mu(\cdot)$ weakly with $\mathrm{t} \rightarrow+\infty$.

Proof. Denote by $f(t ; f(0))$ and $f(t ; \tilde{f}(0))$ two solutions of $(2.2)$ with arbitrary initial values $f(0) \in \mathbb{R}_{+}^{2}$ and $\tilde{f}(0) \in \mathbb{R}_{+}^{2}$, respectively. Define

$$
V(t)=\sum_{i=1}^{2}\left|\ln f_{i}(t ; f(0))-\ln f_{i}(t ; \tilde{f}(0))\right|
$$

By Itô's formula, we have

$$
\begin{aligned}
d V(t)= & \operatorname{sgn}\left(f_{1}(t ; f(0))-f_{1}(t ; \tilde{f}(0))\right)\left[-\left(f_{1}(t ; f(0))-f_{1}(t ; \tilde{f}(0))\right)+\beta_{1}\left(f_{2}(t ; f(0))-f_{2}(t ; \tilde{f}(0))\right)\right] d t \\
& +\operatorname{sgn}\left(f_{2}(t ; f(0))-f_{2}(t ; \tilde{f}(0))\right)\left[\beta_{2}\left(f_{1}(t ; f(0))-f_{1}(t ; \tilde{f}(0))\right)-\left(f_{2}(t ; f(0))-f_{2}(t ; \tilde{f}(0))\right)\right] d t \\
\leqslant & -\left(1-\beta_{2}\right)\left|f_{1}(t ; f(0))-f_{1}(t ; \tilde{f}(0))\right| d t-\left(1-\beta_{1}\right)\left|f_{2}(t ; f(0))-f_{2}(t ; \tilde{f}(0))\right| d t .
\end{aligned}
$$

That is to say,

$$
\begin{aligned}
0 \leqslant \mathbb{E}(V(t)) \leqslant & V(0)-\left(1-\beta_{2}\right) \int_{0}^{t} \mathbb{E}\left|f_{1}(s ; f(0))-f_{1}(s ; \tilde{f}(0))\right| d s \\
& -\left(1-\beta_{1}\right) \int_{0}^{t} \mathbb{E}\left|f_{2}(s ; f(0))-f_{2}(s ; \tilde{f}(0))\right| d s .
\end{aligned}
$$

It then follows from $\mathrm{V}(0)<+\infty$ that

$$
\left(1-\beta_{2}\right) \int_{0}^{t} \mathbb{E}\left|f_{1}(s ; f(0))-f_{1}(s ; \tilde{f}(0))\right| d s+\left(1-\beta_{1}\right) \int_{0}^{t} \mathbb{E}\left|f_{2}(s ; f(0))-f_{2}(s ; \tilde{f}(0))\right| d s \leqslant V(0)<+\infty .
$$

Therefore,

$$
\mathbb{E}\left|f_{i}(t ; f(0))-f_{i}(t ; \tilde{f}(0))\right| \in L^{1}[0, \infty), i=1,2 .
$$

According to (2.2), we have

$$
\begin{aligned}
& \mathbb{E}\left(f_{1}(t)\right)=f_{1}(0)+\int_{0}^{t}\left[\alpha_{1} \mathbb{E}\left(f_{1}(s)\right)-\mathbb{E}\left(f_{1}^{2}(s)\right)+\beta_{1} \mathbb{E}\left(f_{1}(s) f_{2}(s)\right)\right] d s, \\
& \mathbb{E}\left(f_{2}(t)\right)=f_{2}(0)+\int_{0}^{t}\left[\alpha_{2} \mathbb{E}\left(f_{2}(s)\right)-\mathbb{E}\left(f_{2}^{2}(s)\right)+\beta_{2} \mathbb{E}\left(f_{1}(s) f_{2}(s)\right)\right] d s .
\end{aligned}
$$

Consequently, $\mathbb{E}\left(f_{1}(t)\right)$ and $\mathbb{E}\left(f_{2}(t)\right)$ are continuously differentiable. According to (3.1),

$$
\begin{aligned}
& \frac{d \mathbb{E}\left(f_{1}(t)\right)}{d t}=\alpha_{1} \mathbb{E}\left(f_{1}(t)\right)-\mathbb{E}\left(f_{1}^{2}(t)\right)+\beta_{1} \mathbb{E}\left(f_{1}(t) f_{2}(t)\right) \leqslant \alpha_{1} \mathbb{E}\left(f_{1}(t)\right)+\frac{\beta_{1}}{2} \mathbb{E}\left(f_{1}^{2}(t)+f_{2}^{2}(t)\right) \leqslant K_{1}, \\
& \frac{d \mathbb{E}\left(f_{2}(t)\right)}{d t}=\alpha_{2} \mathbb{E}\left(f_{2}(t)\right)-\mathbb{E}\left(f_{2}^{2}(t)\right)+\beta_{2} \mathbb{E}\left(f_{1}(t) f_{2}(t)\right) \leqslant \alpha_{2} \mathbb{E}\left(f_{2}(t)\right)+\frac{\beta_{2}}{2} \mathbb{E}\left(f_{1}^{2}(t)+f_{2}^{2}(t)\right) \leqslant K_{1},
\end{aligned}
$$

where $K_{1}>0$ is a constant. That is to say, $\mathbb{E}\left(f_{1}(t)\right)$ and $\mathbb{E}\left(f_{2}(t)\right)$ are uniformly continuous. It then follows from Barbalat's result [2] that

$$
\lim _{t \rightarrow+\infty} \mathbb{E}\left|f_{i}(t ; f(0))-f_{i}(t ; \tilde{f}(0))\right|=0, i=1,2
$$


Denote by $\mathrm{P}(\mathrm{t}, \mathrm{f}(0), \mathscr{A})$ the probability of event $\{\mathrm{f}(\mathrm{t} ; \mathrm{f}(0)) \in \mathscr{A}\}$ with the initial data $\mathrm{f}(0) \in \mathrm{R}_{+}^{2}$. By virtue of (3.1) and Chebyshev's inequality, the family of transition probabilities $\{p(t, f(0), d x)\}$ is tight $([11,13])$. Denote by $\mathscr{P}\left(\mathbb{R}_{+}^{2}\right)$ all the probability measures on $\mathbb{R}_{+}^{2}$. For $\mathrm{P}_{1}, \mathrm{P}_{2} \in \mathscr{P}$, define

$$
d_{\Lambda}\left(P_{1}, P_{2}\right)=\sup _{\lambda \in \Lambda}\left|\int_{\mathbb{R}_{+}^{2}} \lambda(f) P_{1}(d f)-\int_{\mathbb{R}_{+}^{2}} \lambda(f) P_{2}(d f)\right|,
$$

where

$$
\Lambda=\left\{\lambda: \mathbb{R}_{+}^{2} \rightarrow \mathbb{R}|| \lambda(x)-\lambda(y)|\leqslant\|x-y\|,| \lambda(\cdot) \mid \leqslant 1\right\} .
$$

For arbitrary $\lambda \in \Lambda$ and $t, s>0$, we have

$$
\begin{aligned}
|\mathbb{E} \lambda(f(t+s ; f(0)))-\mathbb{E} \lambda(f(t ; f(0)))| & =\left|\mathbb{E}\left[\mathbb{E}\left(\lambda(f(t+s ; f(0))) \mid \mathcal{F}_{s}\right)\right]-\mathbb{E} \lambda(f(t ; f(0)))\right| \\
& =\left|\int_{\mathbb{R}_{+}^{2}} \mathbb{E} \lambda(f(t ; \tilde{f}(0))) p(s, f(0), d \tilde{f}(0))-\mathbb{E} \lambda(f(t ; f(0)))\right| \\
& \leqslant \int_{\mathbb{R}_{+}^{2}}|\mathbb{E} \lambda(f(t ; \tilde{f}(0)))-\mathbb{E} \lambda(f(t ; f(0)))| p(s, f(0), d \tilde{f}(0)) .
\end{aligned}
$$

In light of (3.17), there exists a $T>0$ such that for $t \geqslant T$,

$$
|\mathbb{E} \lambda(f(t ; \tilde{f}(0)))-\mathbb{E} \lambda(f(t ; f(0)))| \leqslant \mathbb{E}|\lambda(f(t ; \tilde{f}(0)))-\lambda(f(t ; f(0)))| \leqslant \mathbb{E}|f(t ; \tilde{f}(0))-f(t ; f(0))| \leqslant \varepsilon .
$$

Substituting (3.19) into (3.18) leads to

$$
|\mathbb{E} \lambda(f(t+s ; f(0)))-\mathbb{E} \lambda(f(t ; f(0)))| \leqslant \varepsilon, \forall t \geqslant T, s>0 .
$$

It then follows from the arbitrariness of $\lambda$ that

$$
\sup _{\lambda \in \Lambda}|\mathbb{E} \lambda(f(t+s ; f(0)))-\mathbb{E} \lambda(f(t ; f(0)))| \leqslant \varepsilon, \forall t \geqslant T, s>0 .
$$

That is to say,

$$
d_{\wedge}(p(t+s, f(0), \cdot), p(t, f(0), \cdot)) \leqslant \varepsilon \text { for all } t \geqslant T, s>0 .
$$

Hence for any $f(0) \in \mathbb{R}_{+}^{2},\{p(t, f(0), \cdot): t \geqslant 0\}$ is Cauchy in $\mathscr{P}$. Therefore $\{p(t, 0.1, \cdot): t \geqslant 0\}$ is Cauchy in $\mathscr{P}$. It then follows that there exists a unique $\mu(\cdot) \in \mathscr{P}\left(\mathbb{R}_{+}^{2}\right)$ such that

$$
\lim _{t \rightarrow+\infty} d_{\Lambda}(p(t, 0.1, \cdot), \mu(\cdot))=0 .
$$

By (3.17),

$$
\lim _{t \rightarrow+\infty} d_{\Lambda}(p(t, f(0), \cdot), p(t, 0.1, \cdot))=0
$$

Hence

$$
\lim _{t \rightarrow+\infty} d_{\Lambda}(p(t, f(0), \cdot), v(\cdot)) \leqslant \lim _{t \rightarrow+\infty} d_{\Lambda}(p(t, f(0), \cdot), p(t, 0.1, \cdot))+\lim _{t \rightarrow+\infty} d_{\Lambda}(p(t, 0.1, \cdot), \mu(\cdot))=0 .
$$

This completes the proof.

We are now in the position to state and prove our main result.

Theorem 3.6. For model (2.2), 
(i') if $1<\xi_{2}$, then both $\mathrm{f}_{1}$ and $\mathrm{f}_{2}$ are increased, i.e., there is a unique $\mu_{1}(\cdot) \in \mathscr{P}\left(\mathbb{R}_{+}^{2}\right)$, which is ergodic such that

$$
\begin{aligned}
& \lim _{t \rightarrow+\infty} t^{-1} \int_{0}^{t} f_{1}(s) d s=\int_{\mathbb{R}_{+}^{2}} x_{1} \mu_{1}\left(d x_{1}, d x_{2}\right)=\frac{\Delta_{1}}{\Delta}, \\
& \lim _{t \rightarrow+\infty} t^{-1} \int_{0}^{t} f_{2}(s) d s=\int_{\mathbb{R}_{+}^{2}} x_{2} \mu_{1}\left(d x_{1}, d x_{2}\right)=\frac{\Delta_{2}}{\Delta}, \text { a.s. }
\end{aligned}
$$

(ii') if $\xi_{2}<1<\xi_{1}$, then $\mathrm{f}_{2}$ is stagnated a.s., i.e, $\lim _{\mathrm{t} \rightarrow+\infty} \mathrm{f}_{2}(\mathrm{t})=0$, a.s.; at the same time, there is a unique $\mu_{2}(\cdot) \in \mathscr{P}\left(\mathbb{R}_{+}\right)$, which is ergodic such that

$$
\lim _{t \rightarrow+\infty} t^{-1} \int_{0}^{t} f_{1}(s) d s=\int_{\mathbb{R}_{+}^{2}} x_{1} \mu_{2}\left(d x_{1}\right)=b_{1}, \text { a.s. }
$$

(iii') if $\xi_{1}<1$, then both $\mathrm{f}_{1}$ and $\mathrm{f}_{2}$ are stagnated a.s..

Proof. (i'). According to Lemma 3.5, model (2.2) has a unique invariant measure $\mu(\cdot)$. By Corollary 3.4.3 in [7], $\mu(\cdot)$ is strong mixing. An application of Theorem 3.2.6 in [7] gives that $\mu(\cdot)$ is ergodic. Then (3.3.2) in [7] means

$$
\lim _{t \rightarrow+\infty} t^{-1} \int_{0}^{t} f_{i}(s) d s=\int_{\mathbb{R}_{+}^{2}} x_{i} \mu\left(d x_{1}, d x_{2}\right), i=1,2 .
$$

Then the desired assertion follows from Lemma 3.4. The proof of (ii') is similar to that in (i') and hence is omitted. (iii') follows from (iii) in Lemma 3.4.

\section{Numerical simulations}

In this section, we will use Monte Carlo's method (see e.g. [5]) to illustrate the theoretical results. In Fig. 1 , we choose $\alpha_{1}=0.1, \alpha_{2}=0.05, \beta_{1}=0.2, \beta_{2}=0.3$. Then $\Delta=0.94, C_{1}=0.11, C_{2}=0.08$. The only difference between conditions of Fig. 1 (a), Fig. 1 (b), Fig. 1 (c), and Fig. 1 (d) is that the values of $\gamma_{1}$ and $\gamma_{2}$ are different.

(a) In Fig. 1 (a), we let $\gamma_{1}=\gamma_{2}=0$. Then by the results in [21], the positive equilibrium

$$
f^{*}=\left(\frac{C_{1}}{\Delta}, \frac{C_{2}}{\Delta}\right)=\left(\frac{0.11}{0.94}, \frac{0.08}{0.94}\right)=(0.1170,0.0851)
$$

is globally asymptotically stable. See Fig. 1 (a).

(b) In Fig. 1 (b), we choose $\gamma_{1}^{2} / 2=\gamma_{2}^{2} / 2=0.03$. Hence $C_{2}>D_{2}$. According to (i') in Theorem 3.6,

$$
\lim _{t \rightarrow+\infty} t^{-1} \int_{0}^{t} f_{1}(s) d s=\frac{C_{1}-D_{1}}{\Delta}=0.0787, \lim _{t \rightarrow+\infty} t^{-1} \int_{0}^{t} f_{2}(s) d s=\frac{C_{2}-D_{2}}{\Delta}=0.0436 .
$$

See Fig. 1 (b).

(c) In Fig. 1 (c), we let $\gamma_{1}^{2} / 2=0.03, \gamma_{2}^{2} / 2=0.08$. Therefore $\alpha_{1}>\gamma_{1}^{2} / 2$ and $C_{2}<D_{2}$. By virtue of (ii') in Theorem 3.6, $f_{2}$ is stagnated and

$$
\lim _{t \rightarrow+\infty} t^{-1} \int_{0}^{t} f_{1}(s) d s=b_{1}=0.07
$$

See Fig. 1 (c).

(d) In Fig. 1 (d), we set $\gamma_{1}^{2} / 2=0.11, \gamma_{2}^{2} / 2=0.08$. Then $\alpha_{1}>\gamma_{1}^{2} / 2$. It then follows from (iii') in Theorem 3.6 that both $f_{1}$ and $f_{2}$ are stagnated. See Fig. $1(d)$. 


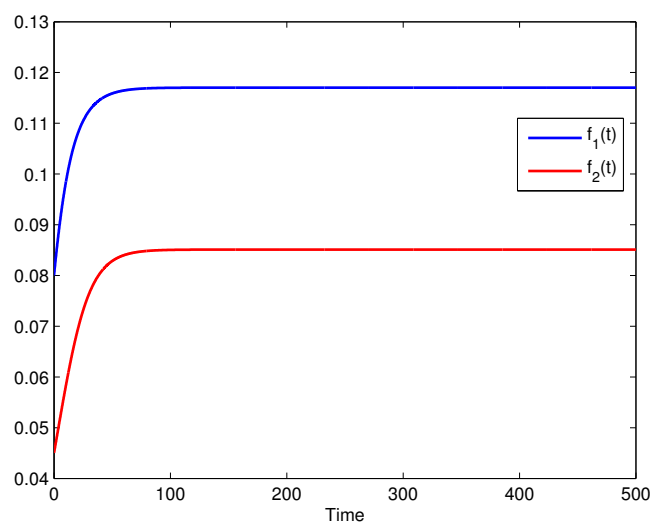

(a)

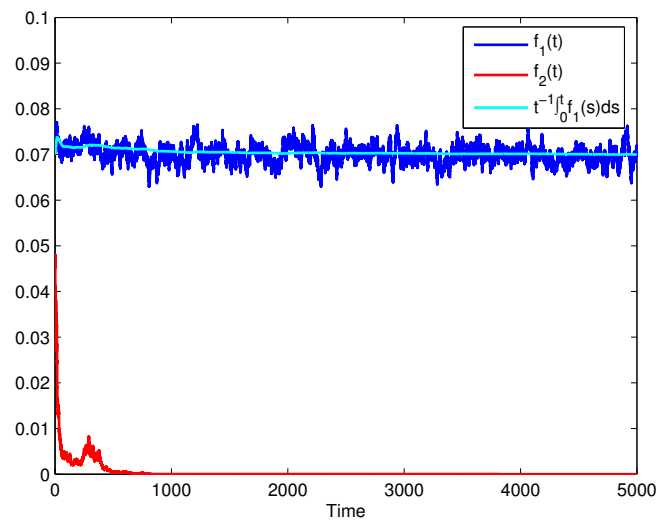

(c)

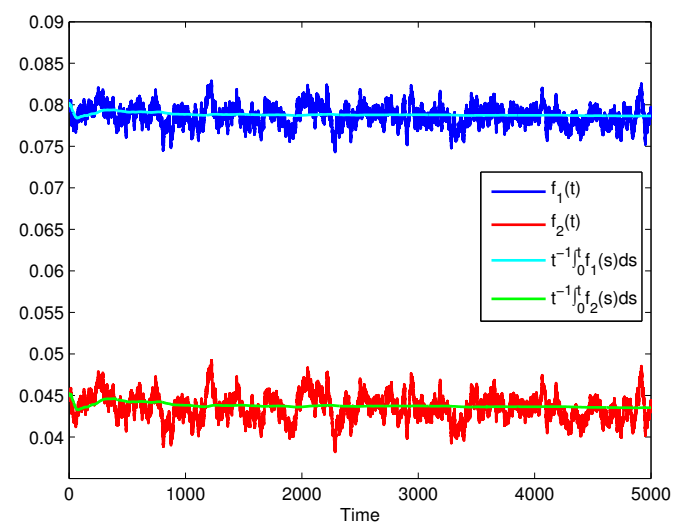

(b)

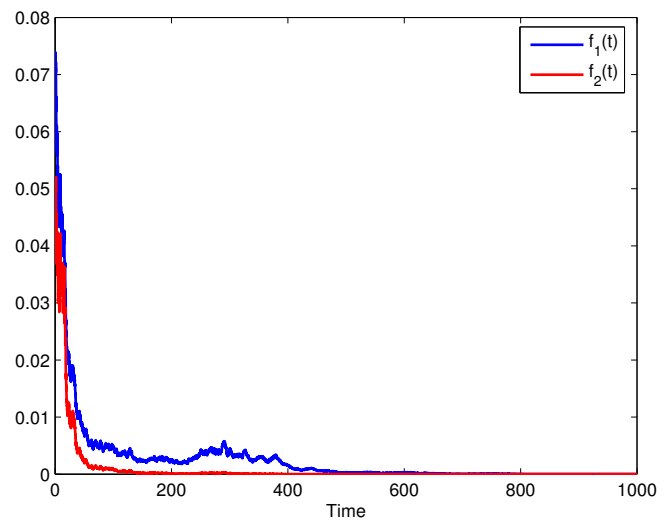

(d)

Figure 1: Solutions of (2.2) for $\alpha_{1}=0.1, \alpha_{2}=0.05, \beta_{1}=0.2, \beta_{2}=0.3$, initial condition $f_{1}(0)=0.08$, $f_{2}(0)=0.05$. (a) is with $\gamma_{1}=\gamma_{2}=0$; (b) is with $\gamma_{1}^{2} / 2=\gamma_{2}^{2} / 2=0.03$; (c) is with $\gamma_{1}^{2} / 2=0.03, \gamma_{2}^{2} / 2=0.08$; (d) is with $\gamma_{1}^{2} / 2=0.11, \gamma_{2}^{2} / 2=0.08$.

\section{Conclusions}

In this paper, we take the random perturbations into account, and propose a stochastic model that describes the interaction between information technology $\left(T_{1}\right)$ and industrial technologies $\left(T_{2}\right)$. Our main result is Theorem 3.6, which establishes sharp sufficient conditions for increase and stagnate of the diffusion rates for both $T_{1}$ and $T_{2}$.

Our results reveal that in some cases, the stochastic perturbations can affect the dynamics of model for $T_{1}$ and $T_{2}$ significantly. In fact, by comparing our results with the work in [21], it is easy to observe that on the one hand, the stochastic perturbations can change the properties of the model significantly, for example, Fig. 1 (a) indicates that the positive equilibrium of model (2.1) is globally asymptotically stable whereas Fig. 1 (c) and (d) show that the diffusion rates in stochastic model (2.2) could be stagnated; on the other hand, stochastic system (2.2) preserves some nice properties of the deterministic model (2.1), for example, Fig. 1 (a) indicates that the diffusion rates in stochastic model (2.2) are increased when the intensities of the stochastic perturbations are not too large.

\section{Acknowledgment}

This research is supported by the National Natural Science Foundation of China (No. 71373123), the Key Project of Philosophy and Social Science Research in Colleges and Universities in Jiangsu Province (No. 2015ZDIXM007). 


\section{References}

[1] X. Bai, L. Lei, A dynamic space-time analysis on the level of integration of informatization and industrialization in China, J. Econ. Geogr., 34 (2014), 52-57. 1

[2] I. Barbălat, Systems dequations differentielles d'osci d'oscillations nonlineaires, Rev. Roumaine Math. Pures Appl., 4 (1959), 267-270. 3

[3] L. Becchetti, D. Bedoya, L. Paganetto, ICT investment, productivity and efficiency: evidence at firm level using a stochastic frontier approach, J. Product. Anal., 20 (2003), 143-167. 1

[4] A. S. Bharadwaj, A resource-based perspective on information technology capability and firm performance: an empirical investigation, MIS Quarterly, 24 (2000), 169-196. 1

[5] N. Bruti-Liberati, E. Platen, Monte Carlo simulation for stochastic differential equations, John Wiley \& Sons, New York, (2010). 4

[6] L. Chen, The Role of Informatization in Industrialization, Huazhong University of Science and Technology, Wuhan, (2011). 1

[7] G. Da Prato, J. Zabczyk, Ergodicity for Infinite Dimensional Systems, Cambridge University Press, Cambridge, (1996). 3

[8] C.-Z. Du, Z.-K. Yang, Research on evaluation of convergence between industrialization and informatization and its development level in China, J. China Univ. Geosci., 15 (2015), 84-97. 1

[9] C. Feng, A Study on the Basic Theory and Solution of integration of Informatization and Industrialization, Wuhan University, Wuhan, (2010). 1

[10] J. Jin, Theoretical system of integration of informatization and industrialization, Informatization Construction, 4 (2009), 9-12. 1

[11] M. Liu, C. Bai, Analysis of a stochastic tri-trophic food-chain model with harvesting, J. Math. Biol., 73 (2016), 597-625. 3

[12] M. Liu, M. Fan, Permanence of stochastic Lotka-Volterra systems, J. Nonlinear Sci., 27 (2017), 425-452. 2

[13] M. Liu, M. Fan, Stability in distribution of a three-species stochastic cascade predator-prey system with time delays, IMA J. Appl. Math., 82 (2017), 396-423. 3

[14] M. Liu, K. Wang, Persistence and extinction in stochastic non-autonomous logistic systems, J. Math. Anal. Appl., 375 (2011), 443-457. 2

[15] M. Liu, K. Wang, Stochastic Lotka-Volterra systems with Lévy noise, J. Math. Anal. Appl., 410 (2014), 750-763. 3

[16] M. Liu, K. Wang, Q. Wu, Survival analysis of stochastic competitive models in a polluted environment and stochastic competitive exclusion principle, Bull. Math. Biol., 73 (2011), 1969-2012. 3.3

[17] H. Lu, Y. Feng, S. Qu, On coordinative development of industrialization and informatization, China Soft Science, 10 (2003), 27-31. 1

[18] X.-R. Mao, C. Yuan, Stochastic Differential Equations with Markovian Switching, Imperial College Press, London, (2006). 3

[19] W. Miao, Transformation of development mode: industrial restructuring and upgrading, Study Times, Beijing, (2011). 1

[20] H. Tong, Research on Evaluation System and Mechanism Analysis of Convergence of Informatization and Industrialization, Beijing University of Posts and Telecommunications, Beijing, (2012). 1

[21] J. Wang, The Mechanism and Performance of Integration of Informatization and Industrialization, Nankai University, Tianjin, (2012). 1, 2, 4, 5

[22] Y.-W. Wang, H. Qin, Spatial pattern and change mechanism analysis on the coupling and coordinating degree of regional informatization and new industrialization in China, J. Econ. Geogr., 34 (2014), 93-100. 1

[23] X.-W. Wang, X. Du, The Fuzzy Evaluation of the Measurement of the Integration of Industrialization and Informationization in China, J. Lanzhou Univ., 42 (2014), 88-97. 1

[24] J. Wu, The road to industrialization, Management World, 8 (2006), 1-7. 1

[25] K. Xie, L. Li, A. Tan, Integration of informatization and industrialization, technical efficiency and convergence, Manage. Rev., 21 (2009), 3-12. 1

[26] K. Xie, J. Xiao, X. Zhou, J. Wu, Quality of convergence between industrialization and informatization in China, Econ. Res. J., 1 (2012), 4-16. 1

[27] L.-P. Yu, Y.-T. Pan, Y.-S. Wu, Study on relationship between industrialization and informatization, China Soft Sci., 1 (2009), 34-40. 1

[28] X. Zhao, Research on the Interaction Mechanism between Informatization and Industrialization, Beijing University of Posts and Telecommunications, Beijing, (2015). 1 